\title{
Modeling and Simulation of Phase Noise Effect on 256-QAM
}

\author{
Bourdillon Odianonsen Omijeh $^{1}$, Ejioeto Evans Ibara ${ }^{2}$ \\ ${ }^{1}$ Department of Electronic \& Computer Engineering, University of Port Harcourt, Port Harcourt, Nigeria \\ ${ }^{2}$ Centre for Information and Telecommunications Engineering, University of Port Harcourt, Port Harcourt, Nigeria \\ Email address: \\ bourdillon.omijeh@uniport.edu.ng (B. O. Omijeh), omijehb@yahoo.com (B. O. Omijeh), ibaraevans@yahoo.com (E. E. Ibara)
}

\section{To cite this article:}

Bourdillon Odianonsen Omijeh, Owanaba Alu Sukubo. Modeling and Simulation of Phase Noise Effect on 256-QAM. International Journal of Wireless Communications and Mobile Computing. Vol. 3, No. 6, 2015, pp. 72-76. doi: 10.11648/j.wcmc.20150306.13

\begin{abstract}
This paper models the effect of phase-noise on a 256-QAM Modulator using Computer Aided Design tool called Matlab/Simulink. By remodeling and varying the noise parameter in the AWGN channel of an expert-system based simulink model, and studying the impact of these variations on the BER of the system, values were recorded for every instance of simulation that was run before and after the addition of the noise. It was found that Phase Noise has an enormous effect on QAM modulators; an effect that grows strong with an increase in the density (version) of the QAM modulators. This implies that higher order QAM are prone to phase noise and hence error, but transmits more data.
\end{abstract}

Keywords: QAM, BER, Phase Noise, PNLD, Noise, AWGN

\section{Introduction}

Phase noise is the fundamental limitation in the performance of a system [1]. Technological advancement often had been driven by the demand for capacity, the limitations and challenges with existing systems, and the struggle to reduce noise effect. A good telecommunication system is measured in terms of its capacity, coverage capability, and the error rate determined at its output. There is more often a trade-off in trying to create a balance between these telecommunication necessities. For instance, an improvement in coverage may result in a noisy signal at the output. Also, to improve the quality of signal at the output of a transmission system, the use of telecommunication system with poorer bandwidth capabilities may just be ideal. The essence of digitizing telecommunication systems, in fact, is to reduce noise and thus the error rate in the received signal [2]. There are different kinds of noise-intrinsic and extrinsic. There are also different kinds of modulation schemes, each with its limitations in terms of noise effects.

\section{Related Works}

QAM is an acronym report demonstrates the effects of phase noise in a 256-QAM system using modeling and for Quadrature Amplitude Modulation. It is one of the many modulation schemes in telecommunication systems. A brief review of works done on the subject of this research is as follows.

\subsection{Theoretical Background}

A Modulation schemes convey message signals by varying a parameter of a carrier signal in accordance to the modulating or message signal. QAM as a modulation scheme does same, but conveys two messages each corresponding to a carrier signal whose amplitude is varied in response to the message being conveyed. According to [3] "QAM mixes two sine waves that are 90 degrees out of phase with one another". This means that, for a QAM scheme there are two carrier signals whose amplitudes are altered in course of transmitting the modulating or message signals. [4] describes QAM as a hybrid modulation scheme in which both "amplitude-shift keying (ASK) and phase-shift keying (PSK)" are varied.

QAMs are predecessors to the phase oriented modulation schemes ranging from BPSK (Binary Phase-Shift Keying), QPSK to 16-PSK. There are different orders of QAM each defined in terms of its prefix value. An increasing order of QAM indicates an increase in the number of information bits that the QAM scheme can convey per symbol. Below in figures $1 \& 2$ [5]" are constellation diagram for 64 QAM and 256 QAM. 


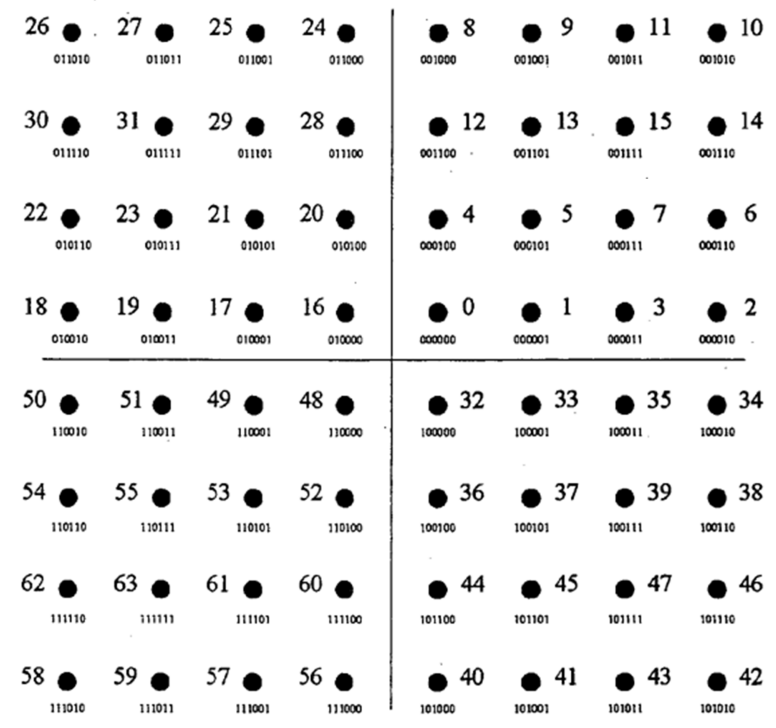

Fig. 1. Constellation diagram for 64-QAM [5].

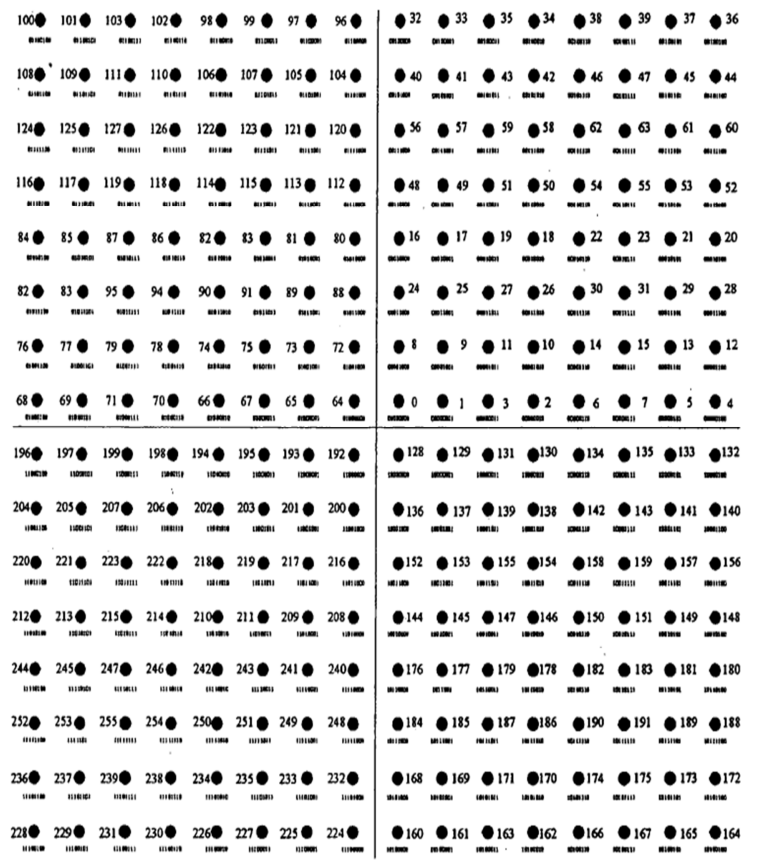

Fig. 2. Constellation diagram for 256-QAM [5].

A run through different QAMs from a lower to higher order indicates that as the points on the constellation gets denser, the distance between them decreases, and the points thus, become more susceptible to noise and other corruption [6]. The result is a higher bit error rate (BER). Using the mathematical expressions in eq 1, Freeman (2006) showed the relationships between the information bits per symbol, the bit error rate (BER), and the Symbol Error Rate (SER). $\log M$ is an excerpt from this expression that represents the $\mathrm{M}$-ary number of the QAMs or its predecessors. Equation (2) represents an expression for computing the precise M-ary or prefix number as described in the work of [7]. It is possible to therefore to determine the information bits per symbol for different QAM schemes as in 2 bits per symbol for 4QAM, 4 bits per symbol for 16QAM, 6 bits per symbol for 64QAM, and 8 bits for 256QAM respectively.

$$
\mathrm{BER}=(1 / \log \mathrm{M}) \times \mathrm{SER}
$$

Where $\mathrm{M}=\mathrm{M}$-ary value prefixing QAM and indicating the version

$$
\mathrm{K}=\log \mathrm{M},
$$

Digital QAM "is constructed using two M-ary baseband signals (called $\mathrm{i}(\mathrm{t})$ and $\mathrm{q}(\mathrm{t})$ ) modulating the two quadrature carriers. For example, in 16-QAM, both $\mathrm{i}(\mathrm{t})$ and $\mathrm{q}(\mathrm{t})$ are 4-ary digital baseband signals, which means each one of them can assume one of four possibilities" [11]. This results in 4 x $4=$ 16 possible carrier symbols shown in the constellation diagrams in Fig. 3 and 4.

\subsection{Effects of Phase Noise}

Noise in transmission is defined as an unintended or unwanted signal introduced into a transmission system. Phase noise is expressed as:

- Loss of sensitivity in radar and communication

- Lack of definition in imagine

- Higher bit error rate (BER) in transmission

The effect of phase noise on an ideal 16-QAM constellation, for instance is as depicted in the figures below. Figure $3 \& 4$ indicates and shows the effect by small amount of phase jitter. Such shifts can be disastrous for more complex constellations than 16-QAM.

Phase Jitter

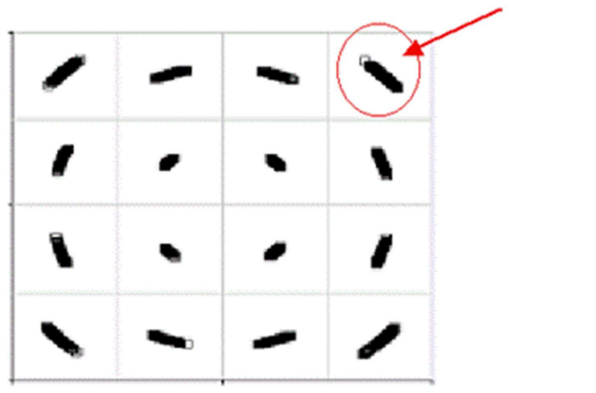

Fig. 3. Phase jilter [5].

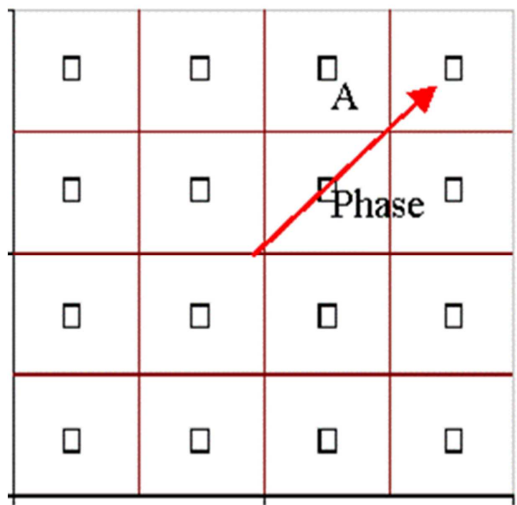

Fig. 4. Phase jilter [5]. 
The amplitude of a phase noise "can be expressed in RMS volts $(\mathrm{Ao} / \sqrt{2})$. Phase noise is typically expressed in units of $\mathrm{dBc} / \mathrm{Hz}$ at various offsets from the carrier frequency. This is usually referred to as Phase Noise Level Density (PNLD)" [8] and can be expressed mathematically as in eq 3 .

$$
\text { PNLD }=-10 \log \mathrm{I} / \mathrm{Io}
$$

Where, $\mathrm{I}=$ noise intensity level in $\mathrm{dB}$, Io $=10^{\wedge}-12$ is a reference noise intensity level

"QAM schemes are enormously efficient in terms of spectrum but its demodulation is difficult particularly in the presence of noise" [9]. Some areas of application of QAM are cable TV, satellites, cellular telephone systems, Wi-Fi, and wireless local-area networks (LANs).

The aim of this work is to model the effect of phase noise on 256-QAM using computer aided design tool Matlab Simulink [10].

\section{Design Methodology}

The study of the phase noise effect is done using an existing model in Simulink. This model was designed to show the effect of phase noise in 256-QAM. First, as a control, it was ensured that the AWGN oriented noise does not have an effect on the modulated signal. This was done by attaching two scatter plot before and after the AWGN channel, and then increasing the value of signal to noise ratio (Es/No in $\mathrm{dB}$ ) in this channel to $100 \mathrm{~dB}$. The Simulink model is as shown in figure 5 below.

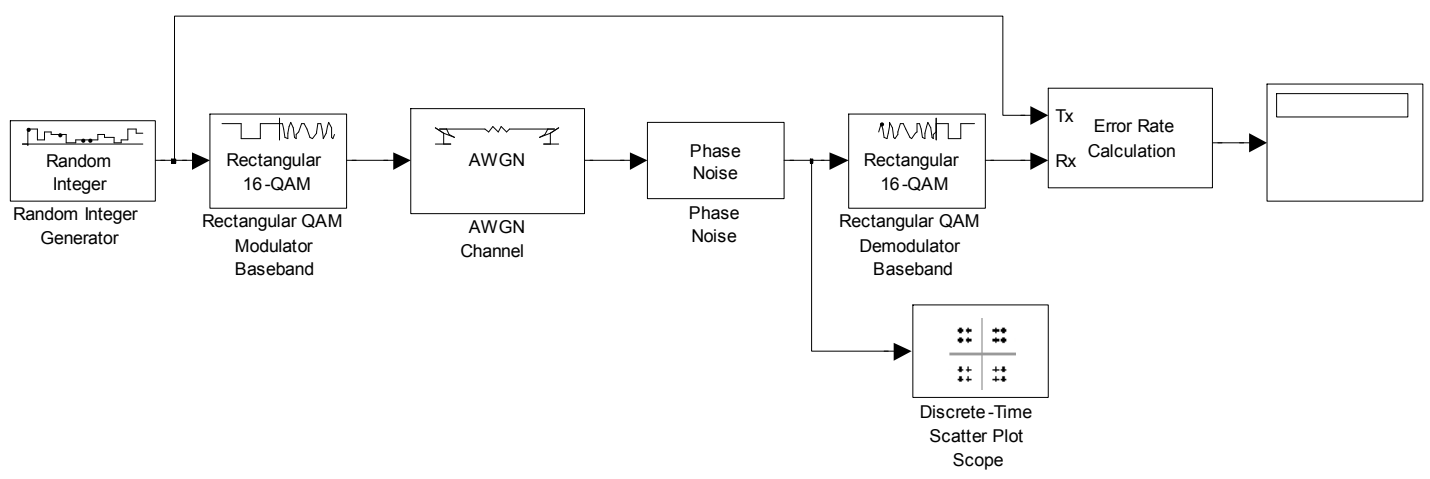

Fig. 5. Phase Noise effect in 256-QAM (model in Simulink).

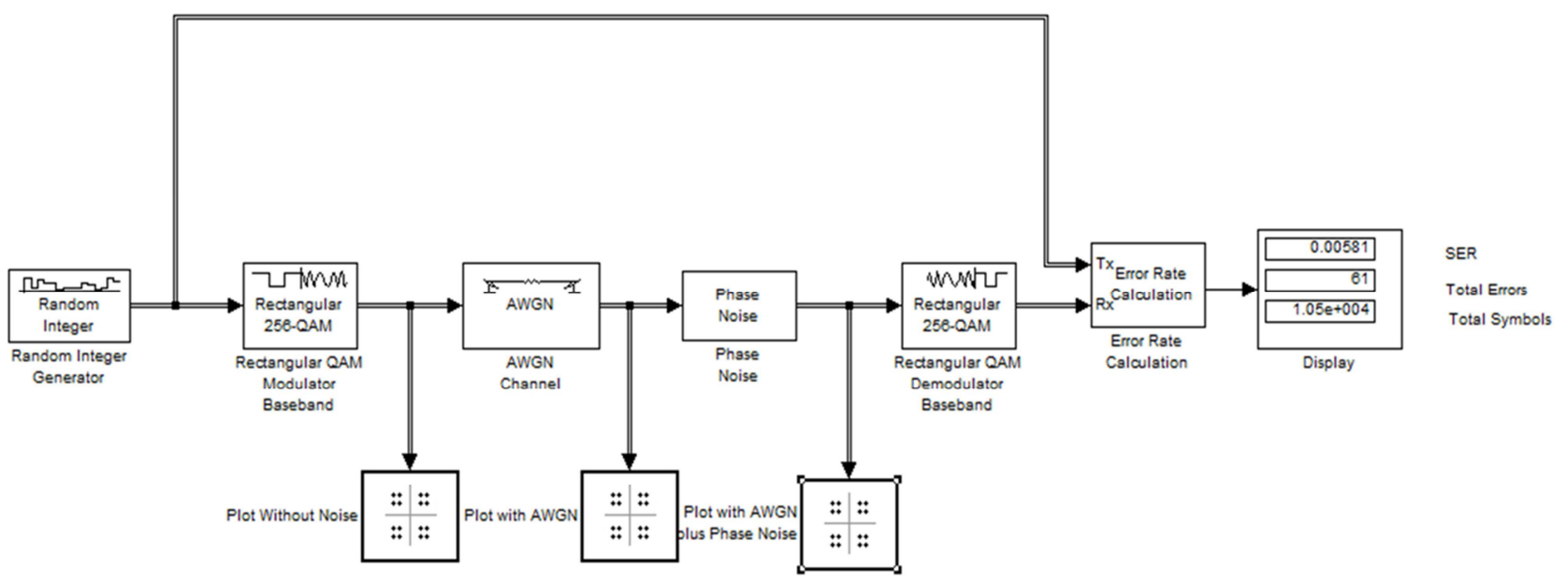

Fig. 6. Phase Noise effect in 256-QAM showing scatter plot before and after AWGN.

The following block sets were used;

- "A baseband 256-QAM modulator

- An additive white Gaussian noise (AWGN) channel

- A source of phase noise

- A baseband 256-QAM demodulator

- An error statistic calculator

- A display icon that shows the error statistics while the simulation runs

- A scatter plot that shows the received signal, including the phase noise"'

\section{Simulation Results and Discussion}

The following parameters were used in each block for the simulation:

\section{1. "Random Integer Generator"}

"Blocks:

a. M-ary number $=256$

b. Initial seed $=12345$ 
c. Sample time $=0.001 \mathrm{~s}$

d. Output status $=$ Frame-based

e. Number of samples per frame $=500$

f. Output data type used $=$ unit 8 "[12].

\section{2. “Rectangular QAM Block”}

a. "M-ary number $=256$

b. Input type $=$ Integer

c. Constellation ordering $=$ Binary

d. Normalization method $=$ Average power

e. Average power $=1$ watts

f. Phase offset $=0 \mathrm{rad}$

g. Output data type = Single".

\section{3. “Rectangular QAM Demodulation Baseband Block"}

a. "M-ary number $=256$

b. Output type $=$ Integer

c. Constellation ordering $=$ Binary

d. Normalization method $=$ Average power

e. Average power $=1$ watts

f. Phase offset $=0 \mathrm{rad}$

g. Output data type = Unit 8".

\section{4. “AWGN Channel”}

a. "Initial seed $=54321$

b. Mode $=$ signal-to-noise ratio $(\mathrm{Es} / \mathrm{No})$

$\mathrm{Es} / \mathrm{No}=100 \mathrm{~dB}$

c. Input signal power $=1$ watts

d. Symbol period $=0.001 \mathrm{~s}$ "

\section{5. "Error Rate Calculation Block"}

a. "Receive delay $=0$

b. Computation delay $=0$

c. Computation mode $=$ entire frame

d. Output data to $=$ port"

\section{6. “Discrete-Time Scatter Plot Scope"}

In $\min X$-axis $=1.5$

Max $X$-axis $=1.5$

Min $\mathrm{Y}$-axis valve $=1.5$

Max $\mathrm{Y}$-axis valve $=1.5$

"In-phase $\mathrm{X}$-axis label $=$ In-phase Amplitude

Quadrature Y-axis label $=$ Quadrature Amplitude

Scope position $=\left[\begin{array}{lll}32 & 306240240\end{array}\right]$

Title $=$ scatter plot".

"The same parameters were set for the three scatter plot scopes we have in the model. They were all set to "open scope at start of simulation". In this case, the scopes open on their own immediately simulation starts".

\section{7. "Configuration Parameters"}

"Simulation time: Start time: 0.0 stop time: 10

Solver $=$ Ode 45

Relative tolerance $=$ ie $-3=0.001$

Absolute tolerance $=$ auto"

\section{8. "Solver Options"}

"Type $=$ variable step

Max step size $=$ auto

Min step size $=$ auto

Initial step size $=$ auto

Zero crossing control = use local settings".

\subsection{Results of the Error Rate Calculation Block}

The results of the Error Rate Calculation Block "helped to give numerical analysis of the effect of the phase noise in the QAM signal for each substituted phase noise density value". This result is represented in table 4.1.

Table 1. Simulation results for a frequency offset of $200 \mathrm{~Hz}$.

\begin{tabular}{llll}
\hline Time & Total Symbol & Total Errors & SER \\
\hline 1 & 1500 & 1414 & 0.9426667 \\
2 & 2500 & 2339 & 0.9356 \\
3 & 3500 & 3261 & 0.9317143 \\
4 & 4500 & 4185 & 0.93 \\
5 & 5500 & 5106 & 0.9283636 \\
6 & 6500 & 6029 & 0.9275385 \\
7 & 7500 & 6968 & 0.9290667 \\
8 & 8500 & 7892 & 0.9284706 \\
9 & 9500 & 8810 & 0.9273684 \\
10 & 10500 & 9728 & 0.9264762 \\
\hline
\end{tabular}

\subsection{Research Findings}

The Error Rate Calculation Block gave varying results as the values of the PNLD were varied from $-20 \mathrm{dBc} / \mathrm{Hz}$ to $-120 \mathrm{dBc} / \mathrm{Hz}$.

Table 2. Result of varied PNLD values with error rate.

\begin{tabular}{llll}
\hline $\begin{array}{l}\text { PNLD } \\
\text { (dBc/Hz) }\end{array}$ & $\begin{array}{l}\text { Number of Symbols } \\
\text { Compared }\end{array}$ & $\begin{array}{l}\text { Number of Errors } \\
\text { that Occurred }\end{array}$ & $\begin{array}{l}\text { Error } \\
\text { Rate }\end{array}$ \\
\hline-20 & 10500 & 10100 & 0.9616 \\
-40 & 10500 & 8828 & 0.8408 \\
-60 & 10500 & 1456 & 0.1387 \\
-80 & 10500 & 0 & 0 \\
-100 & 10500 & 0 & 0 \\
-120 & 10500 & 0 & 0 \\
\hline
\end{tabular}

The first value obtained for PNLD of $-20 \mathrm{dBc} / \mathrm{Hz}$ gave a high rate of error occurrence (of 0.9619). The number of errors that occurred at that noise level was also high (equal to 10100).

As the noise level was reduced to $-40 \mathrm{dBc} / \mathrm{Hz}$ there was a significant reduction (value obtained equals 0.8408 ) in the rate at which error was occurring in the calculation. The number of error calculation at that level was significantly low (value equals 8828) as well.

When the noise level was further reduced to $-60 \mathrm{dBc} / \mathrm{Hz}$, the number of errors that occurred at that point was seen to be: 1456 (which is significantly low when compared to the previous two results).

The same thing applies to the calculated rate at which the error was occurring. The value of the error rate obtained for $60 \mathrm{dBc} / \mathrm{Hz}$ noise level was: 0.1387 .

When the noise level was reduce to $-80 \mathrm{dBc} / \mathrm{Hz}$ and down to $-120 \mathrm{dBc} / \mathrm{Hz}$, zero error was calculated by the Error Rate Calculation Block. 


\section{Conclusion}

From this research it is evident that Quadrature amplitude modulation is seriously affected by phase noise, especially in when the densities are high in the phase noise level. For critical data transmission, a lower order QAM can be used since higher order QAM are prone to higher error rates but transmits more data. Higher data rates are good for transmission but may eventually increase transmission time because of the increase in bit error rate with noise as this may mean that more packets with error will have to be resent.

\section{References}

[1] Dickstein, L. (2012) White Paper. Introduction to Phase Noise in Signal Generators. [Online] Available from: http://www.gigatronics.com/uploads/document/AN-GT140AIntroduction-to-Phase-Noise-in-Signal-Generators.pdf. [Accessed: 10th August 2015].

[2] Biebuma. J.J. Omijeh, B.O, Nathaniel. M.M (2014): Signal Coverage Estimation Model for Microcellular Network Propagation IOSR Journal of Electronics and Communication Engineering (IOSR-JECE), Vol.9, Issue 6 PP 45-53, 2014.

[3] Freeman, R.L. (2008). Technology and Engineering. Radio System Design for Telecommunication. [Online] Available from: http://books.google.com.ng/books?isbn=0470050438.

[4] Kumar, S. (2015) Technology and Engineering. Wireless Communications Fundamental \& Advanced Concepts. [Online] Available from: https://books.google.com.ng/books?isbn=8793102801. [Accessed: 10th August 2015].
[5] Kang, H. \& Kim, J. (2005). Constellation Mapping Apparatus and Method US 20050220205 A1. http://www.google.com/patents/US20050220205. [Accessed: 7th August 2015].

[6] Parihar, G. S. \& Singh, P. (2014) A project Report on Synchronization Techniques for OFDM, 2008-09 Session. http://www.ni.com/white-paper/3896/en/. [Accessed: 7th August 2015].

[7] Ahmad, A. (2003) Data Communication Principles for Fixed and Wireless Networks. [Online] Available from: https://books.google.com.ng/books?isbn=1402073283. [Accessed: 10th August 2015].

[8] Efurumibe, E. L. \& Asiegbu, A. D. (2012) Computer-Based Study of the Effect of Phase Noise in256-Quadrature Amplitude Modulation Using Error-Rate Calculation Block A Comparative Study. Journal of Information Engineering and Applications. [Online] 2(11). P.35. Available from: www.iiste.org. [Accessed: 8th August 2015].

[9] Frenzel, L. (2012) Electronic Design. Understanding Modern Digital Modulation Techniques. [Online]. Available from: $\mathrm{http}$ ://electronicdesign.com/communications/understandingmodern-digital-modulation-techiques. [Accessed: 16th August 2015].

[10] Wetzheng Wang (1996): Communication toolbox user guide, www.mathworks.com.

[11] www.fetweb.ju.edu.jo

[12] www.mathworks.com 